\title{
Double resonance in Sturm-Liouville planar boundary value problems
}

\author{
Andrea Sfecci *
}

\begin{abstract}
We provide some existence results for Sturm-Liouville boundary value problems associated with the planar differential system $J z^{\prime}=g(t, z)+$ $r(t, z)$ where $g$ is suitably controlled by the gradient of two positively homogeneous functions of degree 2 and $r$ is bounded. We study the existence of solutions when a double resonance phenomenon occurs by the introduction of Landesman-Lazer type of conditions. Applications to scalar second order differential equations are given.
\end{abstract}

Keywords: Positively homogeneous planar systems, Sturm-Liouville boundary value problems, Dirichlet problem, shooting method, double resonance, Landesman-Lazer conditions.

Mathematics Subject Classification 2010: 34B15, 37E45, 34A34.

\section{Introduction}

For the scalar equation

$$
x^{\prime \prime}+f(t, x)=0
$$

with perdiodic, Neumann or Dirichlet boundary conditions there have been a lot of researches concerning the existence of solutions under some nonresonance conditions.

The approach to resonance is a delicate problem and the most successful condition have been introduced by Landesman and Lazer, when the nonlinearity asymptotically lies between two eigenvalues of the linear differential equation, see e.g. 7, 8]. In the case of asymmetric nonlinearities we mention [6] for the periodic case and [13 for planar systems (see also the monograph [12] for further informations on this topic).

Even though double resonance phenomenon has been studied dealing with periodic boundary conditions, such a discussion has not been treated for Dirichlet and Neumann boundary conditions yet. In this paper we are going to present

${ }^{*}$ Dipartimento di Scienze Matematiche, Università Politecnica delle Marche, Via Brecce Bianche 1, 60131 Ancona - Italy. email: sfecci@dipmat.univpm.it 
some existence results at double resonance for equation 1.1 when $f$ satisfies

$$
\begin{aligned}
& 0<\nu_{1} \leq \liminf _{x \rightarrow-\infty} \frac{f(t, x)}{x} \leq \limsup _{x \rightarrow-\infty} \frac{f(t, x)}{x} \leq \nu_{2}, \\
& 0<\mu_{1} \leq \liminf _{x \rightarrow+\infty} \frac{f(t, x)}{x} \leq \limsup _{x \rightarrow+\infty} \frac{f(t, x)}{x} \leq \mu_{2}
\end{aligned}
$$

(see Theorem 4.3 below).

In such a situation the nonlinearity $f$ "mimes" an asymmetric oscillator

$$
x^{\prime \prime}+\mu x^{+}-\nu x^{-}=0
$$

where $x^{+}=(|x|+x) / 2$ and $x^{-}=(|x|-x) / 2$. The previous scalar differential equation can be studied as a planar system of the type

$$
J z^{\prime}=\nabla V(z), \quad z \in \mathbb{R}^{2}
$$

where $J=\left(\begin{array}{cc}0 & -1 \\ 1 & 0\end{array}\right)$ is the standard symplectic matrix and $V: \mathbb{R}^{2} \rightarrow \mathbb{R}$ is a positively homogeneous $C^{1}$-function of degree 2 , i.e. such that

$$
0<V(\lambda z)=\lambda^{2} V(z), \quad \text { for every } \lambda>0, z \neq 0 \text {. }
$$

For this reason, boundary value problems related to 1.4 present a particular interest in literature, see e.g. [1, 11, 14, 25] and the references therein.

In relation with the scalar second order differential equation 1.1 , the Dirichlet boundary conditions $x(0)=x(T)=0$ (DBC), the Neumann boundary conditions $x^{\prime}(0)=x^{\prime}(T)=0$ (NBC) and the mixed boundary conditions $x^{\prime}(0)=x(T)=0$ (MBC) can be collected all together in a unique class of problems when we pass to consider planar systems as in 1.4. Indeed, we can ask a solution $z(t)=(x(t), y(t))$ to start and arrive at some points belonging to two lines in the plane:

$$
z(0) \in l_{S}, \quad z(T) \in l_{A},
$$

where $l_{S}$ is the starting line and $l_{A}$ is the arrival line. In particular (DBC) is equivalent to the case $l_{S}=l_{A}=\{z=(x, y) \mid x=0\}$, (NBC) is equivalent to the case $l_{S}=l_{A}=\{z=(x, y) \mid y=0\}$ and (MBC) is equivalent to the case $l_{S}=\{z=(x, y) \mid y=0\}$ and $l_{A}=\{z=(x, y) \mid x=0\}$.

In [1, 14] the following class of problems, obtained as a perturbation of (1.4), is treated:

$$
\left\{\begin{array}{l}
J z^{\prime}=\nabla V(z)+p(t, z), \\
z(0) \in l_{S}, \quad z(T) \in l_{A},
\end{array}\right.
$$

where, for briefness, we say $p$ is bounded and continuous. 
Recalling that the unperturbed system 1.4 has an isochronous center of minimal period $\tau_{V}$, and borrowing the definition from [1, we say that the unperturbed problem

$$
\left\{\begin{array}{l}
J z^{\prime}=\nabla V(z), \\
z(0) \in l_{S}, \quad z(T) \in l_{A} .
\end{array}\right.
$$

is resonant if it has at least one nontrivial solution. As in the periodic case, if problem (1.7) is not resonant then a perturbed problem as in 1.6 admit a solution, cf. 14. Conversely, if the unperturbed problem (1.7) is resonant, then the existence of a solution to problems as in 1.6 is ensured assuming an additional condition: in [1] the introduction of a Landesman-Lazer type of assumption provides an existence result. In these notes we continue the study performed in [1, 14 by Boscaggin, Fonda and Garrione. In particular, we are going to consider the wider class of problems

$$
\left\{\begin{array}{l}
J z^{\prime}=g(t, z)+p(t, z), \\
z(0) \in l_{S}, \quad z(T) \in l_{A},
\end{array}\right.
$$

where the function $g$ is controlled by two positively homogeneous functions of degree $2, V_{1} \leq V_{2}$ : More precisely, $g$ satisfies

$$
g(t, z)=(1-\gamma(t, z)) \nabla V_{1}(z)+\gamma(t, z) \nabla V_{2}(z),
$$

where $\gamma:[0, T] \times \mathbb{R}^{2} \rightarrow \mathbb{R}$, with $0 \leq \gamma \leq 1$ and $p:[0, T] \times \mathbb{R}^{2} \rightarrow \mathbb{R}^{2}$ is sublinear with respect to the second variable.

The study of existence of solutions to problem 1.8$)$ is related to the study of perturbed asymmetric oscillators, e.g. differential equations as $x^{\prime \prime}+\mu x^{+}-$ $\nu x^{-}+g(x)=e(t)$ where $g$ and $e$ are bounded continuous functions. In particular, (1.8) includes the previously mentioned scalar differential equation (1.1) with $f$ obeying to $(1.2)$ and $(1.3)$, as a particular case. Such a type of problems presents a wide literature, see e.g. [2, 3, 4, 5, 6, 10, 11, 12, 18, 19, 20, 21, for a nonexhaustive bibliography, apologizing for unavoidable missing references.

Systems as in 1.8 have been investigated in [13, by Fonda and Garrione dealing with periodic boundary conditions (see also [9, 15]). In the periodic setting, the existence of solutions can be ensured if there exists a positive integer $k$ such that

$$
\frac{T}{k+1} \leq \tau_{V_{2}} \leq \tau_{V_{1}} \leq \frac{T}{k},
$$

where $\tau_{V_{1}}$ and $\tau_{V_{2}}$ are the periods of the solutions of system (1.4) choosing respectively $V=V_{1}$ and $V=V_{2}$.

In such a situation we can distinguish three situations: nonresonance, when we have the strict inequalities in (1.9); simple resonance, when we have a strict inequality and an equality in 1.9 ; double resonance, when both equalities hold in (1.9). In presence of resonance we need to add additional assumptions, e.g. of Landesman-Lazer type, as suggested in [13] (see also [6, 7, 8, 23, 24] for related results). 
In this paper, dealing with system $(1.8)$, we investigate all the three situations: nonresonance, simple resonance and double resonance, which will be treated in Section 3. The situations differ depending on the position of the value $T$ in 1.8 with respect to a resonance set which will be introduced in 2.19.

The paper is organized as follows. In Section 2 we present some preliminary results: in Section 2.1 some properties of the autonomous system (1.4) are listed borrowing some notations from 1, 14, then in Section 2.2 we add a first perturbation presenting some properties of solutions of systems $(1.8)$ in the the semi-autonomous case $r \equiv 0$. We present the main Theorems 3.2 (nonresonance), 3.7 (simple resonance) and 3.8 (double resonance) in the successive Sections 3.1 3.2 and 3.3. Finally, in Section 4 , we present the applications of our theorems in the case of scalar equations (1.1) with an asymmetric nonlinearity, cf. Theorem 4.3 .

Let us here introduce some notations. We will denote by $|\cdot|$ the Euclidean norm in $\mathbb{R}^{2}$ and we will use the complex notation for polar coordinates in the plane, i.e. $z=(x, y)=\rho e^{i \vartheta}=(\rho \cos \vartheta, \rho \sin \vartheta)$. Moreover, in order to well define the angle $\vartheta$ when we pass to polar coordinates, we will consider functions $z: I \rightarrow \mathbb{R}^{2}$ such that $z(t) \neq(0,0)$ for every $t \in I$. For briefness we call them never-zero functions.

\section{Preliminaries}

\subsection{An autonomous isochronous planar system}

In this section we recall some notations and contents from [1, 14. Let us consider the planar system

$$
J z^{\prime}=\nabla V(z), \quad z=(x, y) \in \mathbb{R}^{2},
$$

where $J=\left(\begin{array}{cc}0 & -1 \\ 1 & 0\end{array}\right)$ is the standard symplectic matrix and $V: \mathbb{R}^{2} \rightarrow \mathbb{R}$ is a $C^{1}$-function which is positively homogeneous of degree 2 , i.e.

$$
0<V(\lambda z)=\lambda^{2} V(z), \quad \text { for every } \lambda>0, z \neq 0 .
$$

Let us recall the validity of the Euler's formula: $\langle\nabla V(z) \mid z\rangle=2 V(z)$ for every $z \in \mathbb{R}^{2}$.

System (2.1) is an isochronous center of minimal period

$$
\tau_{V}=\int_{0}^{2 \pi} \frac{d \theta}{2 V(\cos \theta, \sin \theta)}
$$

and all the solutions have the form $z(t)=C \varphi_{V}(t+\tau)$, with $C \geq 0$ and $\tau \in\left[0, \tau_{V}\right)$, where $\varphi_{V}$ is a fixed nontrivial solution to 2.1). Without loss of generality we assume $V\left(\varphi_{V}(t)\right) \equiv \frac{1}{2}$ and $\varphi_{V}(0)=\left(0, y_{0}\right)$ with $y_{0}>0$. 
Let us consider the following boundary condition

$$
z(0) \in l_{S}, \quad z(T) \in l_{A},
$$

where $l_{S}$ and $l_{A}$ (" $S$ " stands for starting, " $A$ " for arrival) are lines through the origin of slope $\zeta_{S}$ and $\zeta_{A}$, respectively. We mean that a line through the origin has slope $\zeta \in(-\pi / 2, \pi / 2]$ if it can be parametrized as $l: \mathbb{R} \rightarrow \mathbb{R}^{2}$, $l(s)=s(\cos \zeta, \sin \zeta)$.

For later purpose, let us introduce

$$
\Delta \zeta= \begin{cases}\zeta_{S}-\zeta_{A} & \text { if } \zeta_{S}>\zeta_{A} \\ \zeta_{S}-\zeta_{A}+\pi & \text { if } \zeta_{S} \leq \zeta_{A},\end{cases}
$$

which is the smallest positive angle a solution covers moving from $l_{S}$ to $l_{A}$, cf. Figure 1 (remember that solutions rotate clockwise).

Let us now recall some notations:

- $\tau_{0, V}$ is the least nonnegative time such that $\varphi_{V}\left(\tau_{0, V}\right) \in l_{S}$,

- $\tau_{1, V}$ is the least positive time such that $\varphi_{V}\left(\tau_{0, V}+\tau_{1, V}\right) \in l_{A}$,

- $\sigma_{1, V}$ is the least nonnegative time such that $\varphi_{V}\left(\tau_{0, V}+\tau_{1, V}+\sigma_{1, V}\right) \in l_{S}$,

- $\tau_{2, V}$ is the least positive time such that $\varphi_{V}\left(\tau_{0, V}+\tau_{1, V}+\sigma_{1, V}+\tau_{2, V}\right) \in l_{A}$,

- $\sigma_{2, V}$ is the least nonnegative time such that $\varphi_{V}\left(\tau_{0, V}+\tau_{1, V}+\sigma_{1, V}+\tau_{2, V}+\right.$ $\left.\sigma_{2, V}\right) \in l_{S}$.

Notice that, by definition,

$$
\tau_{V}=\tau_{1, V}+\sigma_{1, V}+\tau_{2, V}+\sigma_{2, V}
$$

and in particular, as in 2.3), we have

$$
\begin{gathered}
\tau_{1, V}=\int_{\zeta_{S}-\Delta \zeta}^{\zeta_{S}} \frac{d \theta}{2 V(\cos \theta, \sin \theta)}, \quad \tau_{2, V}=\int_{\zeta_{S}+\pi-\Delta \zeta}^{\zeta_{S}+\pi} \frac{d \theta}{2 V(\cos \theta, \sin \theta)}, \\
\sigma_{1, V}=\int_{\zeta_{S}-\pi}^{\zeta_{S}-\Delta \zeta} \frac{d \theta}{2 V(\cos \theta, \sin \theta)}, \quad \sigma_{2, V}=\int_{\zeta_{S}}^{\zeta_{S}+\pi-\Delta \zeta} \frac{d \theta}{2 V(\cos \theta, \sin \theta)},
\end{gathered}
$$

As a consequence, if $l_{S}$ and $l_{A}$ coincide, then $\sigma_{1, V}=\sigma_{2, V}=0$.

In order to distinguish the two semi-lines which $l_{A}$ and $l_{S}$ consist of respectively, let us introduce the following notations (cf. Figure 1)

$$
\begin{array}{ll}
l_{S}^{1} \ni \varphi_{V}\left(\tau_{0, V}\right), & l_{A}^{1} \ni \varphi_{V}\left(\tau_{0, V}+\tau_{1, V}\right), \\
l_{S}^{2} \ni \varphi_{V}\left(\tau_{0, V}+\tau_{1, V}+\sigma_{1, V}\right), & l_{A}^{2} \ni \varphi_{V}\left(\tau_{0, V}+\tau_{1, V}+\sigma_{1, V}+\tau_{2, V}\right) .
\end{array}
$$

The problem

$$
\left\{\begin{array}{l}
J z^{\prime}=\nabla V(z), \\
z(0) \in l_{S}, \quad z(T) \in l_{A},
\end{array}\right.
$$




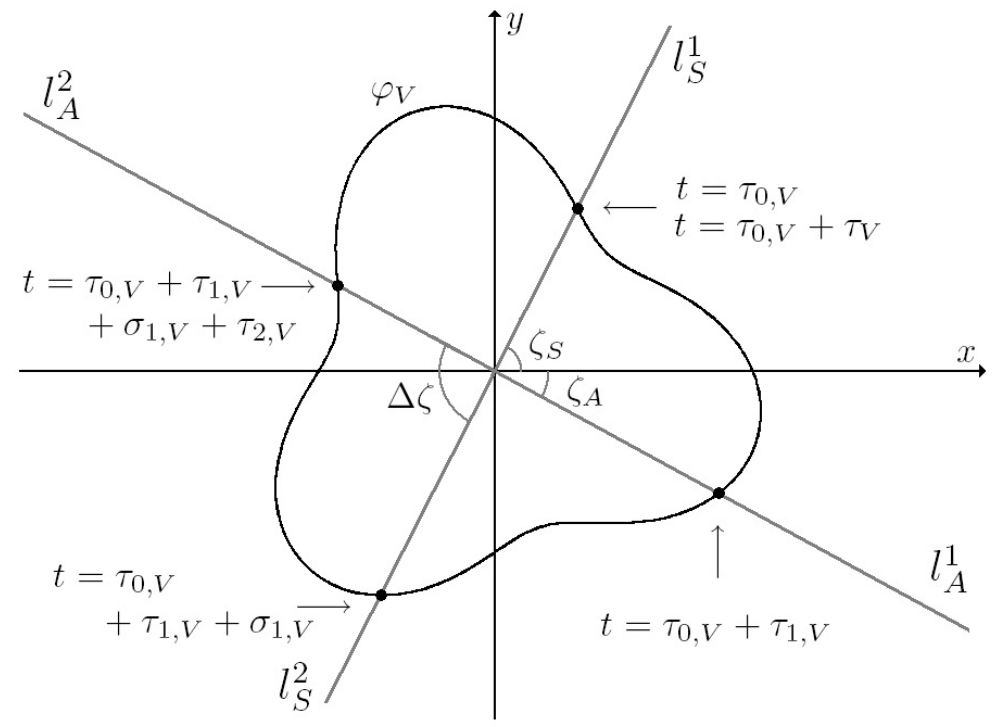

Figure 1: The notation of Section 2.1, a sketch in the case $\zeta_{A}<\zeta_{S}$.

is said to be resonant if it admits nontrivial solutions. Such a situation occurs if and only if $T$ satisfies at least one of the following identities, for a certain $k \in \mathbb{N}$,

$$
\begin{aligned}
T & =k \tau_{V}+\tau_{1, V}, \\
T & =k \tau_{V}+\tau_{1, V}+\sigma_{1, V}+\tau_{2, V}, \\
T & =k \tau_{V}+\tau_{2, V}, \\
T & =k \tau_{V}+\tau_{2, V}+\sigma_{2, V}+\tau_{1, V} .
\end{aligned}
$$

and a nontrivial solution is given by

$$
\begin{aligned}
\varphi_{V}\left(t+\tau_{0, V}\right) & \text { if } 2.10 \text { or } 2.11 \text { holds, } \\
\varphi_{V}\left(t+\tau_{0, V}+\tau_{1, V}+\sigma_{1, V}\right) & \text { if } 2.12 \text { or } 2.13 \text { holds. }
\end{aligned}
$$

Indeed, we can distinguish different starting semilines and arrival semilines:

2.10 holds $\Rightarrow$ the solution starts from $l_{S}^{1}$ and arrives on $l_{A}^{1}$,

(2.11) holds $\Rightarrow$ the solution starts from $l_{S}^{1}$ and arrives on $l_{A}^{2}$,

(2.12) holds $\Rightarrow$ the solution starts from $l_{S}^{2}$ and arrives on $l_{A}^{2}$, 2.13 holds $\Rightarrow$ the solution starts from $l_{S}^{2}$ and arrives on $l_{A}^{1}$. 


\subsection{Introducing a perturbation in the energy}

We now focus our attention on the qualitative properties of solutions to the boundary value problem

$$
\left\{\begin{array}{l}
J z^{\prime}=g(t, z), \\
z(0) \in l_{S}, \quad z(T) \in l_{A},
\end{array}\right.
$$

where the function $g:[0, T] \times \mathbb{R}^{2} \rightarrow \mathbb{R}^{2}$ is suitably controlled by two Hamiltonians $V_{1}$ and $V_{2}$ as in the previous section. More precisely, we introduce the following assumption.

Assumption 2.1. Assume that there exists a $L^{2}$-Carathéodory function $\gamma$ : $[0, T] \times \mathbb{R}^{2} \rightarrow[0,1]$ such that

$$
g(t, z)=(1-\gamma(t, z)) \nabla V_{1}(z)+\gamma(t, z) \nabla V_{2}(z)
$$

where $V_{1} \leq V_{2}$ are two positively homogeneous $C^{1}$-functions as in 2.2.

Let us now borrow the notations of the previous section, cf. (2.6), and we define the values

$$
\begin{aligned}
& \tau_{V_{2}}=\tau_{1, V_{2}}+\sigma_{1, V_{2}}+\tau_{2, V_{2}}+\sigma_{2, V_{2}},
\end{aligned}
$$

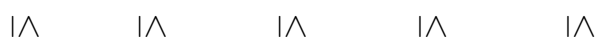

$$
\begin{aligned}
& \tau_{V_{1}}=\tau_{1, V_{1}}+\sigma_{1, V_{1}}+\tau_{2, V_{1}}+\sigma_{2, V_{1}} .
\end{aligned}
$$

Let us set

$$
\begin{array}{ll}
\alpha_{2 k}=\min \left\{a_{2 k}^{1}, a_{2 k}^{2}\right\}, & a_{2 k}^{1}=k \tau_{V_{2}}+\tau_{1, V_{2}}, \\
& a_{2 k}^{2}=k \tau_{V_{2}}+\tau_{2, V_{2}} ; \\
\beta_{2 k}=\max \left\{b_{2 k}^{1}, b_{2 k}^{2}\right\}, & b_{2 k}^{1}=k \tau_{V_{1}}+\tau_{1, V_{1}}, \\
& b_{2 k}^{2}=k \tau_{V_{1}}+\tau_{2, V_{1}} ; \\
\alpha_{2 k+1}=\min \left\{a_{2 k+1}^{1}, a_{2 k+1}^{2}\right\}, & a_{2 k+1}^{1}=k \tau_{V_{2}}+\tau_{1, V_{2}}+\tau_{2, V_{2}}+\sigma_{1, V_{2}}, \\
& a_{2 k+1}^{2}=k \tau_{V_{2}}+\tau_{1, V_{2}}+\tau_{2, V_{2}}+\sigma_{2, V_{2}} ; \\
\beta_{2 k+1}=\max \left\{b_{2 k+1}^{1}, b_{2 k+1}^{2}\right\}, & b_{2 k+1}^{1}=k \tau_{V_{1}}+\tau_{1, V_{1}}+\tau_{2, V_{1}}+\sigma_{1, V_{1}}, \\
& b_{2 k+1}^{2}=k \tau_{V_{1}}+\tau_{1, V_{1}}+\tau_{2, V_{1}}+\sigma_{2, V_{1}} ;
\end{array}
$$

and define the intervals $I_{j}=\left[\alpha_{j}, \beta_{j}\right], j \in \mathbb{N}$. Notice that all the intervals are well ordered in the following sense: $\alpha_{j}<\alpha_{j+1}$ and $\beta_{j}<\beta_{j+1}$ for every $j \in \mathbb{N}$,

We introduce the resonance set

$$
\mathcal{I}=\bigcup_{j \in \mathbb{N}} I_{j}=\bigcup_{j \in \mathbb{N}}\left[\alpha_{j}, \beta_{j}\right],
$$

the interior of $\mathcal{I}$, denoted by $\stackrel{\circ}{\mathcal{I}}$, and

$$
\widetilde{\mathcal{I}}=\bigcup_{j \in \mathbb{N}}\left(\alpha_{j}, \beta_{j}\right) .
$$


Notice that $\widetilde{\mathcal{I}} \subseteq \stackrel{\circ}{\mathcal{I}} \subseteq \mathcal{I}$ and so $\partial \mathcal{I} \subseteq \partial \widetilde{\mathcal{I}}$.

In this paper we are going to treat the following situations:

- Nonresonance: $T \notin \mathcal{I}$, i.e. $\exists \kappa \in \mathbb{N}$ such that $\beta_{\kappa}<T<\alpha_{\kappa+1}$, or $T<\alpha_{0}$.

- Simple resonance: $T \in \partial \mathcal{I}$, i.e. $\exists \kappa \in \mathbb{N}$ such that $T=\alpha_{\kappa}$ or $T=\beta_{\kappa}$.

- Double resonance: $T \in \partial \widetilde{\mathcal{I}} \backslash \partial \mathcal{I}$, i.e. $\exists \kappa \in \mathbb{N}$ such that $T=\beta_{\kappa}=\alpha_{\kappa+1}$.

Introducing polar coordinates $z=(x, y)=\rho e^{i \vartheta}$, the angular velocity of a never-zero solution of 2.16) is given by

$$
\begin{aligned}
-\vartheta^{\prime}( & t)=\frac{\left\langle J z^{\prime}(t) \mid z(t)\right\rangle}{|z(t)|^{2}} \\
= & \left(1-\gamma(t, z(t)) \frac{\left\langle\nabla V_{1}(z(t)) \mid z(t)\right\rangle}{|z(t)|^{2}}+\gamma(t, z(t)) \frac{\left\langle\nabla V_{2}(z(t)) \mid z(t)\right\rangle}{|z(t)|^{2}}\right. \\
= & 2\left(1-\gamma(t, z(t)) V_{1}(\cos \vartheta(t), \sin \vartheta(t))+2 \gamma(t, z(t)) V_{2}(\cos \vartheta(t), \sin \vartheta(t))\right.
\end{aligned}
$$

so that we obtain

$$
0<2 V_{1}(\cos \vartheta(t), \sin \vartheta(t)) \leq-\vartheta^{\prime}(t) \leq 2 V_{2}(\cos \vartheta(t), \sin \vartheta(t)) .
$$

By the previous computation, recalling (2.3), 2.7) and the notation introduced in 2.8, a never-zero solution of 2.16)

moving from $l_{S}^{1}$ to $l_{A}^{1}$ spends a time $\Delta t_{1} \in\left[\tau_{1, V_{2}}, \tau_{1, V_{1}}\right]$,

moving from $l_{A}^{1}$ to $l_{S}^{2}$ spends a time $\Delta t_{2} \in\left[\sigma_{1, V_{2}}, \sigma_{1, V_{1}}\right]$,

moving from $l_{S}^{2}$ to $l_{A}^{2}$ spends a time $\Delta t_{3} \in\left[\tau_{2, V_{2}}, \tau_{2, V_{1}}\right]$,

moving from $l_{A}^{2}$ to $l_{S}^{1}$ spends a time $\Delta t_{4} \in\left[\sigma_{2, V_{2}}, \sigma_{2, V_{1}}\right]$,

completes a rotation around the origin in a time $\Delta t \in\left[\tau_{V_{2}}, \tau_{V_{1}}\right]$.

\section{Main results}

\subsection{Nonresonance}

In this section we consider the boundary value problem

$$
\left\{\begin{array}{l}
J z^{\prime}=g(t, z)+p(t, z), \\
z(0) \in l_{S}, \quad z(T) \in l_{A},
\end{array}\right.
$$

where $g:[0, T] \times \mathbb{R}^{2} \rightarrow \mathbb{R}^{2}$ satisfies Assumption 2.1 and $p$ satisfies

Assumption 3.1. The function $p:[0, T] \times \mathbb{R}^{2} \rightarrow \mathbb{R}^{2}$ is a Carathéodory function such that, for every compact set $K \subset \mathbb{R}^{2},|p(t, z)| \leq \ell_{K}(t)$, for a.e. $t \in[0, T]$ and $z \in K$, for a suitable $\ell_{K} \in L^{2}(0, T)$. Moreover,

$$
\lim _{|z| \rightarrow \infty} \frac{p(t, z)}{z}=0
$$

uniformly for almost every $t \in[0, T]$. 
Let us introduce some notations. We will denote by $\Phi: \mathbb{R} \times \mathbb{R}^{2} \rightarrow \mathbb{R}^{2}$ the flux of system

$$
J z^{\prime}=g(t, z)+p(t, z),
$$

i.e. $\Phi\left(\cdot, z_{0}\right)$ is the solution $z$ of $(3.3)$ such that $z(0)=\Phi\left(0, z_{0}\right)=z_{0}{ }_{1}^{1}$ We will also consider polar coordinates associated to never-zero solutions $z=\Phi\left(\cdot, z_{0}\right)$ :

$$
\Phi\left(t, z_{0}\right)=\mathscr{R}\left(t, z_{0}\right)\left(\cos \Theta\left(t, z_{0}\right), \sin \Theta\left(t, z_{0}\right)\right) .
$$

For definiteness, we will need to specify the value $\Theta\left(0, z_{0}\right)$ or we will preferably consider the covered-angle function

$$
\Delta \Theta\left(t, z_{0}\right)=\Theta\left(0, z_{0}\right)-\Theta\left(T, z_{0}\right) \geq 0 .
$$

In this section we are going to prove the following result which generalizes [14, Theorem 3.1]:

Theorem 3.2 (Nonresonance). Consider problem (3.1), where g satisfies Assumption 2.1 and p satisfies Assumption 3.1. If $T \notin \mathcal{I}$, where $\mathcal{I}$ is the resonance set introduced in (2.19), then there exists at least one solution of (3.1).

Proof. If $T \notin \mathcal{I}$, then we have $T \in\left(\beta_{j-1}, \alpha_{j}\right)$ for a certain $j \in \mathbb{N}$ (set $\left.\beta_{-1}=0\right)$. In particular we can find a small $\epsilon>0$ such that

$$
T \in\left(\beta_{j-1}+(2 j-1) \epsilon, \alpha_{j}-(2 j+1) \epsilon\right) .
$$

Introducing polar coordinates $z=\rho e^{i \vartheta}$, the angular velocity of solutions of 3.3 is given by

$$
-\vartheta^{\prime}(t)=\frac{\langle g(t, z(t))+p(t, z(t)) \mid z(t)\rangle}{|z(t)|^{2}}
$$

and we can compute

$$
2 V_{1}(\cos \vartheta(t), \sin \vartheta(t))+e(t) \leq-\vartheta^{\prime}(t) \leq 2 V_{2}(\cos \vartheta(t), \sin \vartheta(t))+e(t) .
$$

where

$$
e(t)=\frac{\langle p(t, z(t)) \mid z(t)\rangle}{|z(t)|^{2}} .
$$

Then, from $2.22-(2.26)$, for every $\epsilon>0$ we can find $R_{1}>0$ such that a solution of 3.3$)$, satisfying $|z(t)| \geq R_{1}$ for every $t \in[0, T]$, rotates clockwise and

moving from $l_{S}^{1}$ to $l_{A}^{1}$ spends a time $\Delta t_{1} \in\left(\tau_{1, V_{2}}-\epsilon, \tau_{1, V_{1}}+\epsilon\right)$, moving from $l_{A}^{1}$ to $l_{S}^{2}$ spends a time $\Delta t_{2} \in\left(\sigma_{1, V_{2}}-\epsilon, \sigma_{1, V_{1}}+\epsilon\right)$,

moving from $l_{S}^{2}$ to $l_{A}^{2}$ spends a time $\Delta t_{3} \in\left(\tau_{2, V_{2}}-\epsilon, \tau_{2, V_{1}}+\epsilon\right)$,

moving from $l_{A}^{2}$ to $l_{S}^{1}$ spends a time $\Delta t_{4} \in\left(\sigma_{2, V_{2}}-\epsilon, \sigma_{2, V_{1}}+\epsilon\right)$,

completes a rotation around the origin in a time

$$
\Delta t \in\left(\tau_{V_{2}}-4 \epsilon, \tau_{V_{1}}+4 \epsilon\right) .
$$

\footnotetext{
${ }^{1}$ We can assume, without loss of generality, the uniqueness of the solutions to the Cauchy problems. Indeed, by standard arguments, all the results in this paper can be obtained with a limit procedure introducing a sequence of approximating nonlinearities having such a uniqueness property.
} 
Then, by the elastic property we can prove the existence of $R_{2}>R_{1}$ such that if a solution of (3.3) satisfies $|z(\bar{t})| \geq R_{2}$ for a certain $\bar{t} \in[0, T]$, then $|z(t)| \geq R_{1}$ for every $t \in[0, T]$. E.g., we can adopt a guiding curve method as in [16, 17, 22. Lemma 4.2 in [16] works well in our situation.

Summing up, we can conclude with the following result.

Proposition 3.3. For every $j \in \mathbb{N}$ and every $\epsilon>0$, there exists $R_{j, \epsilon}>R_{2}$ such that

- if $z^{1} \in l_{S}^{1}$ and $\left|z^{1}\right| \geq R_{j, \epsilon}$ then the solution $\Phi\left(\cdot, z^{1}\right)$ covers the angle $j \pi+\Delta \zeta$ in a time $\tau \in\left(a_{j}^{1}-(2 j+1) \epsilon, b_{j}^{1}+(2 j+1) \epsilon\right)$.

- if $z^{2} \in l_{S}^{2}$ and $\left|z^{2}\right| \geq R_{j, \epsilon}$ then the solution $\Phi\left(\cdot, z^{2}\right)$ covers the angle $j \pi+\Delta \zeta$ in a time $\tau \in\left(a_{j}^{2}-(2 j+1) \epsilon, b_{j}^{2}+(2 j+1) \epsilon\right)$.

As a consequence we can rewrite the previous proposition as follows, cf. Figure 2 (set $R_{-1, \epsilon}=0$ for definiteness).

Remark 3.4. Fix $R>\max \left\{R_{j-1, \epsilon}, R_{j, \epsilon}\right\}$. Setting $z^{1}=R\left(\cos \zeta_{S}, \sin \zeta_{S}\right) \in l_{S}^{1}$ and $z^{2}=-R\left(\cos \zeta_{S}, \sin \zeta_{S}\right) \in l_{S}^{2}$. Then

$$
\begin{aligned}
& \Delta \Theta\left(T, z^{1}\right) \in((j-1) \pi+\Delta \zeta, j \pi+\Delta \zeta) . \\
& \Delta \Theta\left(T, z^{2}\right) \in((j-1) \pi+\Delta \zeta, j \pi+\Delta \zeta) .
\end{aligned}
$$

In particular, $\Phi\left(T, z^{1}\right)$ and $\Phi\left(T, z^{2}\right)$ belongs to different connected components of $\mathbb{R}^{2} \backslash l_{A}$.

By standard argument (cf. Figure 22) the curve $\eta:[-R, R] \rightarrow \mathbb{R}^{2}$ defined as $\eta(\sigma)=\Phi\left(T, \sigma\left(\cos \zeta_{S}, \sin \zeta_{S}\right)\right)$ intersects the line $l_{A}$ for a certain $\bar{\sigma}$ and the proof of Theorem 3.2 is given.

\subsection{Simple resonance}

In this section we consider the case $T \in \partial \mathcal{I}$, where $\mathcal{I}$ was introduced in $(2.19)$. In such a situation $\beta_{j-1}<T=\alpha_{j}$ or $\beta_{j-1}=T<\alpha_{j}$ for a certain $j \in \mathbb{N}$. In order to obtain the existence of a solution to (3.1) we need to introduce a Landesman-Lazer type of condition. We can distinguish the following cases:

$$
\begin{gathered}
T=\alpha_{j}=a_{j}^{1}<a_{j}^{2}, \\
T=\alpha_{j}=a_{j}^{2}<a_{j}^{1}, \\
T=\alpha_{j}=a_{j}^{1}=a_{j}^{2} ; \\
T=\beta_{j-1}=b_{j-1}^{1}>b_{j-1}^{2}, \\
T=\beta_{j-1}=b_{j-1}^{2}>b_{j-1}^{1}, \\
T=\beta_{j-1}=b_{j-1}^{1}=b_{j-1}^{2} .
\end{gathered}
$$




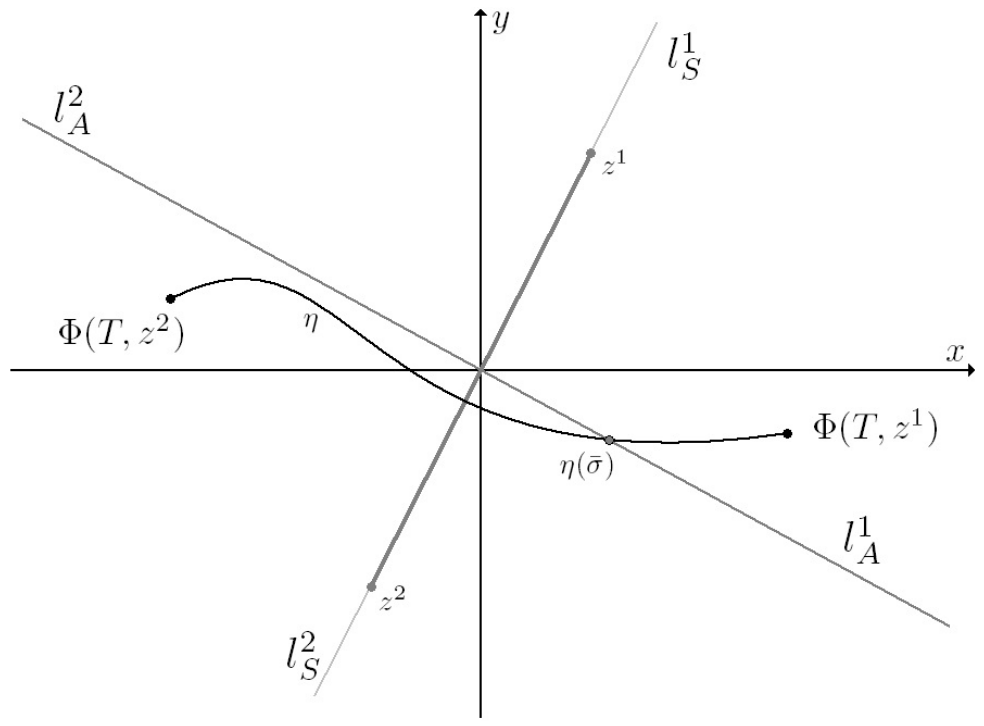

Figure 2: A sketch on Remark 3.4 and an idea of the proof of Theorem 3.2

Let us introduce the following functions, where $G(t, z)=g(t, z)+p(t, z)$ :

$$
\begin{array}{r}
\mathcal{J}^{-}(\theta)=\int_{0}^{T} \liminf _{(\lambda, \omega) \rightarrow(+\infty, \theta)}\left[\left\langle G\left(t, \lambda \varphi_{V_{1}}(t+\omega)\right) \mid \varphi_{V_{1}}(t+\omega)\right\rangle-\right. \\
\left.-2 \lambda V_{1}\left(\varphi_{V_{1}}(t+\omega)\right)\right] d t \\
\mathcal{J}^{+}(\theta)=\int_{0}^{T} \limsup _{(\lambda, \omega) \rightarrow(+\infty, \theta)}\left[\left\langle G\left(t, \lambda \varphi_{V_{2}}(t+\omega)\right) \mid \varphi_{V_{2}}(t+\omega)\right\rangle-\right. \\
\left.-2 \lambda V_{2}\left(\varphi_{V_{2}}(t+\omega)\right)\right] d t .
\end{array}
$$

The Landesman-Lazer type of assumption we need to require can be summarized as follows. 
Assumption 3.5 (Landesman-Lazer type of assumptions).

$$
\begin{aligned}
& \text { If R1 holds, assume } \mathcal{J}^{+}\left(\tau_{0, V_{2}}\right)<0 \text {, } \\
& \text { if (R2) holds, assume } \mathcal{J}^{+}\left(\tau_{0, V_{2}}+\tau_{1, V_{2}}+\sigma_{1, V_{2}}\right)<0 \text {, } \\
& \text { if }(\overline{\mathrm{R} 3}) \text { holds, assume } \mathcal{J}^{+}\left(\tau_{0, V_{2}}\right)<0 \text { and } \\
& \mathcal{J}^{+}\left(\tau_{0, V_{2}}+\tau_{1, V_{2}}+\sigma_{1, V_{2}}\right)<0 \\
& \text { if (R4) holds, assume } \mathcal{J}^{-}\left(\tau_{0, V_{1}}\right)>0 \text {, } \\
& \text { if R5 holds, assume } \mathcal{J}^{-}\left(\tau_{0, V_{1}}+\tau_{1, V_{1}}+\sigma_{1, V_{1}}\right)>0 \text {, } \\
& \text { if (R6) holds, assume } \mathcal{J}^{-}\left(\tau_{0, V_{1}}\right)>0 \text { and } \\
& \mathcal{J}^{-}\left(\tau_{0, V_{1}}+\tau_{1, V_{1}}+\sigma_{1, V_{1}}\right)>0 .
\end{aligned}
$$

In the case of simple resonance only one of the alternatives $(\mathrm{R} 1)-(\mathrm{R} 6)$ is verified, and so we need to check only one of the alternatives in Assumption 3.5.

Remark 3.6 (A comparison with [1]). In [1] the case $V_{1}=V_{2}$ has been studied. In such a situation $a_{j}^{i}=b_{j}^{i}$ for every $j \in \mathbb{N}$ and $i \in\{1,2\}$. In particular Theorem 2.1 and Theorem 2.3-2 in [1] treats the case " $j$ is even", while Theorem 2.3-1 and Theorem 2.3-3 the case " $j$ is odd".

E.g., in [1, Theorem 2.1] the situations (17),(18),(19) or (20) correspond respectively to (R1), (R4), (R3) and (R6).

The existence result is the following.

Theorem 3.7 (Simple resonance). Consider problem (3.1), where $p$ satisfies Assumption 3.1 and $g$ satisfies Assumption 2.1. If $T \in \partial \mathcal{I}$, where $\mathcal{I}$ was introduced in (2.19), then there exists at least one solution of (3.1) provided that Landesman-Lazer Assumption 3.5 is fulfilled.

Proof. Let us start assuming the validity of (R1), in particular $\beta_{j-1}<T=\alpha_{j}$.

Let us consider $z^{2} \in l_{S}^{2}$ and the solution $\Phi\left(\cdot, z^{2}\right)$ of $(3.3)$. By Proposition 3.3 . if $\left|z^{2}\right|$ is sufficiently large then

$$
\Delta \Theta\left(T, z^{2}\right) \in((j-1) \pi+\Delta \zeta, j \pi+\Delta \zeta) .
$$

In fact, $b_{j-1}^{2}<T<a_{j}^{2}$ holds.

Let us now consider $z^{1} \in l_{S}^{1}$ and the solution $\Phi\left(\cdot, z^{1}\right)$ of (3.3). Arguing similarly, if $\left|z^{1}\right|$ is sufficiently large then

$$
\Delta \Theta\left(T, z^{1}\right) \in((j-1) \pi+\Delta \zeta,(j+1) \pi+\Delta \zeta) .
$$

Notice that the interval is larger since $T=a_{j}^{1}$. We need to prove that the situation $\Delta \Theta\left(T, z^{1}\right) \in[j \pi+\Delta \zeta,(j+1) \pi+\Delta \zeta)$ is forbidden. Once this claim is proved, we will obtain the existence of $R>0$ with the property explained in Remark 3.4 thus permitting us to conclude the proof of the theorem as in the previous section. 
We argue by contradiction and suppose the existence of a sequence $\left(z_{n}^{0}\right)_{n} \subset$ $l_{S}^{1}$, with $\left|z_{n}^{0}\right| \rightarrow \infty$, such that

$$
\Delta \Theta\left(T, z_{n}^{0}\right) \in[j \pi+\Delta \zeta,(j+1) \pi+\Delta \zeta) .
$$

Set $z_{n}(t)=\Phi\left(t, z_{n}^{0}\right)$ and introduce the sequence

$$
w_{n}=\frac{z_{n}}{\left\|z_{n}\right\|_{\infty}}=\frac{\Phi\left(\cdot, z_{n}^{0}\right)}{\left\|\Phi\left(\cdot, z_{n}^{0}\right)\right\|_{\infty}}
$$

consisting of solutions to

$$
\left\{\begin{array}{l}
J w_{n}^{\prime}=\left(1-\Gamma_{n}(t)\right) \nabla V_{1}\left(w_{n}\right)+\Gamma_{n}(t) \nabla V_{2}\left(w_{n}\right)+\frac{p\left(t, w_{n}(t)\left\|z_{n}\right\|_{\infty}\right)}{\left\|z_{n}\right\|_{\infty}}, \\
w_{n}(0) \in l_{S}^{1}
\end{array}\right.
$$

where $\Gamma_{n}(t)=\gamma\left(t, w_{n}(t)\left\|z_{n}\right\|_{\infty}\right)$. Hence, $\left(w_{n}\right)_{n}$ is bounded in $H^{1}(0, T)$, so that up to a subsequence it converges uniformly and weakly in $H^{1}(0, T)$ to a certain non-trivial function $w$ satisfying $w(0) \in l_{S}^{1}$. Moreover, the sequence $\left(\Gamma_{n}\right)_{n}$ is bounded in $L^{2}$ and converges weakly to a certain $\Gamma \in L^{2}(0, T)$, up to a subsequence. The sequence is contained in the closed convex subset $\{q \in$ $L^{2}(0, T) \mid 0 \leq q(t) \leq 1$ a.e. in $\left.[0, T]\right\}$ so that we have also $0 \leq \Gamma \leq 1$ a.e. in $[0, T]$. Then, passing to the weak limit in 3.19 we get

$$
\left\{\begin{array}{l}
J w^{\prime}=(1-\Gamma(t)) \nabla V_{1}(w)+\Gamma(t) \nabla V_{2}(w), \\
w(0) \in l_{S}^{1} .
\end{array}\right.
$$

We claim that $\Gamma \equiv 1$ a.e. in $[0, T]$.

From (3.17), setting $w_{n}^{0}=w_{n}(0)=\frac{z_{n}^{0}}{\left\|z_{n}\right\|_{\infty}}$ and using polar coordinates $w_{n}=$ $\varrho_{n} e^{i \theta_{n}}$,

we get $\theta_{n}(0)-\theta_{n}(T) \in[j \pi+\Delta \zeta,(j+1) \pi+\Delta \zeta)$. Hence, setting $w=\varrho e^{i \theta}$,

$$
\theta(0)-\theta(T) \in[j \pi+\Delta \zeta,(j+1) \pi+\Delta \zeta]
$$

so that there exists $\bar{t} \in[0, T]$ such that $\theta(0)-\theta(\bar{t})=j \pi+\Delta \zeta$. Hence, integrating

$$
-\frac{\theta^{\prime}(t)}{2 V_{2}(\cos \theta(t), \sin \theta(t))} \leq 1
$$

in the interval $[0, \bar{t}]$ we get

$$
\int_{\zeta_{S}-(j \pi+\Delta \zeta)}^{\zeta_{S}} \frac{d \theta}{2 V_{2}(\cos \theta, \sin \theta)}=a_{j}^{1} \leq \bar{t}
$$

By hypothesis $a_{j}^{1}=T$, hence $\bar{t}=T$ holds.

We have proved that $w(T) \in l_{A}$ and $w$ cover the angle $j \pi+\Delta \zeta$ in the interval $[0, T]$. 
Let us now parametrize $w$ using the polar coordinates induced by $\varphi_{V_{2}}$ :

$$
w(t)=r(t) \varphi_{V_{2}}(t+\omega(t)) .
$$

A standard computation provide

$$
\begin{gathered}
r^{\prime}(t)=-r(t)(1-\Gamma(t))\left\langle\nabla V_{1}\left(\varphi_{V_{2}}(t+\omega(t))\right) \mid \varphi_{V_{2}}^{\prime}(t+\omega(t))\right\rangle, \\
\omega^{\prime}(t)=(1-\Gamma(t))\left(2 V_{1}\left(\varphi_{V_{2}}(t+\omega(t))\right)-1\right) .
\end{gathered}
$$

Notice that $\omega(0)=\tau_{0, V_{2}}$ and $\omega(T)=\tau_{0, V_{2}}+a_{j}^{1}-T=\tau_{0, V_{2}}$, so that

$$
0=\int_{0}^{T} \omega^{\prime}(t) d t=\int_{0}^{T}(1-\Gamma(t))\left(2 V_{1}\left(\varphi_{V_{2}}(t+\omega(t))\right)-1\right) d t .
$$

Recalling that $V_{1} \leq V_{2}$, we get $2 V_{1}\left(\varphi_{V_{2}}\right)-1 \leq 2 V_{2}\left(\varphi_{V_{2}}\right)-1=0$ so that

$$
(1-\Gamma(t))\left(2 V_{1}\left(\varphi_{V_{2}}(t+\omega(t))\right)-1\right) \leq 0 \quad \text { a.e. in }[0, T] .
$$

Hence, from (3.26), we necessarily have

$$
(1-\Gamma(t))\left(2 V_{1}\left(\varphi_{V_{2}}(t+\omega(t))\right)-1\right)=0 \quad \text { a.e. in }[0, T],
$$

in particular $\omega \equiv \tau_{0, V_{2}}$ a.e. in $[0, T]$.

Let us now focus our attention on the radial velocity formula (3.24). We are going to prove that $r^{\prime} \equiv 0$ almost everywhere in $[0, T]$. Let us consider $t_{0} \in[0, T]$ such that $\Gamma\left(t_{0}\right)<1$ (the situation is trivial if $\Gamma\left(t_{0}\right)=1$ ). By (3.27) we necessarily have $2 V_{1}\left(\varphi_{V_{2}}\left(t_{0}+\omega\left(t_{0}\right)\right)\right)=1$. Recalling that $V_{2} \geq V_{1}$ and $2 V_{2}\left(\varphi_{V_{2}}\right) \equiv 1$, we find that $t_{0}$ is a minimum of the function $\mathcal{V}(t)=V_{2}\left(\varphi_{V_{2}}(t+\right.$ $\omega(t)))-V_{1}\left(\varphi_{V_{2}}(t+\omega(t))\right)$, precisely $\mathcal{V}\left(t_{0}\right)=0$, so that $\mathcal{V}^{\prime}\left(t_{0}\right)=0$. Being $V_{2}$ constant along $\varphi_{V_{2}}$ we get $\left.\frac{d}{d t} V_{1}\left(\varphi_{V_{2}}(\cdot+\omega)\right)\right|_{t=t_{0}}=0$ and consequently $\left\langle\nabla V_{1}\left(\varphi_{V_{2}}\left(t_{0}+\omega\left(t_{0}\right)\right)\right) \mid \varphi_{V_{2}}^{\prime}\left(t_{0}+\omega\left(t_{0}\right)\right)\right\rangle=0$, giving $r^{\prime}\left(t_{0}\right)=0$.

We have proved that $w=C \varphi_{V_{2}}\left(t+\tau_{0, V_{2}}\right)$ for a certain constant $C>0$, so that $\Gamma \equiv 1$ a.e. in $[0, T]$.

Let us consider again the sequence $\left(w_{n}\right)_{n}$ introduced in (3.18) and the polar coordinates $w_{n}=\varrho_{n} e^{i \theta_{n}}$. We have

$$
\int_{\theta_{n}(T)}^{\theta_{n}(0)} \frac{d \theta}{2 V_{2}(\cos \theta, \sin \theta)} \geq T,
$$

for large indexes $n$ by the validity of (3.17): indeed, the integral provides the time spent by a solution of the system $J z^{\prime}=\nabla V_{2}(z)$ to cover the angular sector between $\theta_{n}(0)=\zeta_{S}$ and $\theta_{n}(T) \leq \zeta_{S}-(\pi j+\Delta \zeta)$, while $T$ is the time spent to cover the (not larger) angular sector between $\zeta_{S}$ and $\zeta_{S}-(\pi j+\Delta \zeta)$.

The angular speed of $w_{n}$ is given by

$$
\begin{aligned}
-\theta_{n}^{\prime}(t)= & \frac{\left\langle J w_{n}^{\prime}(t) \mid w_{n}(t)\right\rangle}{\left|w_{n}(t)\right|^{2}}=\frac{\left\langle J z_{n}^{\prime}(t) \mid z_{n}(t)\right\rangle}{\left|z_{n}(t)\right|^{2}} \\
= & 2 V_{2}\left(\cos \theta_{n}(t)\right. \\
& \left., \sin \theta_{n}(t)\right) \\
& \quad+\frac{\left\langle g\left(t, z_{n}(t)\right)+p\left(t, z_{n}(t)\right)-\nabla V_{2}\left(z_{n}(t)\right) \mid z_{n}(t)\right\rangle}{\left|z_{n}(t)\right|^{2}}
\end{aligned}
$$


thus giving

$$
\int_{\theta_{n}(T)}^{\theta_{n}(0)} \frac{d \theta}{2 V_{2}(\cos \theta, \sin \theta)}=T+\int_{0}^{T} \frac{\left\langle\mathcal{G}\left(t, z_{n}(t)\right) \mid z_{n}(t)\right\rangle}{2 V_{2}\left(z_{n}(t)\right)} d t,
$$

where $\mathcal{G}(t, z)=g(t, z)+p(t, z)-\nabla V_{2}(z)$. By (3.28), we obtain

$$
\mathcal{X}_{n}:=\int_{0}^{T} \frac{\left\langle\mathcal{G}\left(t, z_{n}(t)\right) \mid z_{n}(t)\right\rangle}{2 V_{2}\left(z_{n}(t)\right)} \geq 0
$$

for sufficiently large indexes $n$. We parametrize the solutions $z_{n}$ in the polar coordinates induced by $\varphi_{V_{2}}$, i.e. we set

$$
z_{n}(t)=r_{n}(t) \varphi_{V_{2}}\left(t+\omega_{n}(t)\right) .
$$

So, we obtain

$$
\mathcal{X}_{n}=\int_{0}^{T} \frac{\left\langle\mathcal{G}\left(t, r_{n}(t) \varphi_{V_{2}}\left(t+\omega_{n}(t)\right)\right) \mid \varphi_{V_{2}}\left(t+\omega_{n}(t)\right)\right\rangle}{r_{n}(t)}
$$

From 3.18 and recalling that $w_{n} \rightarrow w=C \varphi_{V_{2}}\left(t+\tau_{0, V_{2}}\right)$ uniformly, we have $\frac{r_{n}(t)}{\left\|z_{n}\right\|_{\infty}} \rightarrow C$ and $\omega_{n} \rightarrow \tau_{0, V_{2}}$. Then

$$
\begin{aligned}
0 & \leq \limsup _{n \rightarrow+\infty}\left\|z_{n}\right\|_{\infty} \mathcal{X}_{n} \\
& \leq \int_{0}^{T} \limsup _{n \rightarrow+\infty} \frac{\left\langle\mathcal{G}\left(t, r_{n}(t) \varphi_{V_{2}}\left(t+\omega_{n}(t)\right)\right) \mid \varphi_{V_{2}}\left(t+\omega_{n}(t)\right)\right\rangle}{\frac{r_{n}(t)}{\left\|z_{n}\right\|_{\infty}}} d t \\
& \leq \frac{1}{C} \int_{0}^{T} \limsup _{(\lambda, \omega) \rightarrow\left(+\infty, \tau_{0, V_{2}}\right)}\left\langle\mathcal{G}\left(t, \lambda \varphi_{V_{2}}(t+\omega)\right) \mid \varphi_{V_{2}}(t+\omega)\right\rangle d t
\end{aligned}
$$

The last inequality, using (3.14, can be rewritten as $\mathcal{J}^{+}\left(\tau_{0, V_{2}}\right) \geq 0$ which contradicts Assumption 3.5 .

We have proved the theorem if (R1) holds.

Let us now spend few words in order to explain how to adapt the proof in the other situations.

Assume R2. In such a situation the role of $l_{S}^{1}$ and $l_{S}^{2}$ is switched. So, the "good estimate" in (3.15) is easily obtained for $z^{1} \in l_{S}^{1}$, with $\left|z^{1}\right|$ sufficiently large, while the "bad estimate" (3.16) occurs treating $z^{2} \in l_{S}^{2}$. In this case, we assume by contradiction the existence of a sequence $\left(z_{n}^{0}\right)_{n} \subset l_{S}^{2}$, with $\left|z_{n}^{0}\right| \rightarrow \infty$, satisfying (3.17). The proof can be plainly adapted, but we underline the main differences: the starting angle $\Theta\left(0, z_{n}^{0}\right)=\zeta_{S}$ is replaced by $\Theta\left(0, z_{n}^{0}\right)=\zeta_{S}+\pi$ and the constant $a_{j}^{1}$ is replaced by $a_{j}^{2}$. In particular, 3.22 becomes

$$
\int_{\zeta_{S}+\pi-(j \pi+\Delta \zeta)}^{\zeta_{S}+\pi} \frac{d \theta}{2 V_{2}(\cos \theta, \sin \theta)}=a_{j}^{2} \leq \bar{t} .
$$

Finally, the limit function is now $w(t)=C \varphi_{V_{2}}\left(t+\tau_{0, V_{2}}+\tau_{1, V_{2}}+\sigma_{1, V_{2}}\right)$. 
Assume now (R3). Under this hypothesis, we get the "bad estimates" (3.16) both for $z_{0} \in l_{S}^{1}$ and $z_{0} \in l_{S}^{2}$ with $\left|z_{0}\right|$ large so that the proof is a glueing of cases $(\mathrm{R} 1)$ and $(\mathrm{R} 2)$. The same reasoning holds for $(\mathrm{R} 6)$ : the proof will follow by the ones of cases ( $\mathrm{R} 4)$ and $(\mathrm{R} 5)$ we are going to provide.

Let us consider (R4). The validity of (3.15) is given when we treat solutions $\Phi\left(\cdot, z_{0}\right)$ of $\sqrt{3.3}$ with $z_{0} \in l_{S}^{2}$ with $\left|z_{0}\right|$ sufficiently large. However, solutions $\Phi\left(\cdot, z_{0}\right)$ of 3.3 with $z_{0} \in l_{S}^{1}$ satisfies (no more 3.16 , but)

$$
\Delta \Theta\left(T, z_{0}\right) \in((j-2) \pi+\Delta \zeta, j \pi+\Delta \zeta) .
$$

and we need to forbid the situation $\Delta \Theta\left(T, z_{0}\right) \in((j-2) \pi+\Delta \zeta,(j-1) \pi+\Delta \zeta]$. Arguing as above, we can consider a diverging sequence $\left(z_{n}^{0}\right)_{n} \subset \ell_{S}^{1}$ such that

$$
\Delta \Theta\left(T, z_{n}^{0}\right) \in((j-2) \pi+\Delta \zeta,(j-1) \pi+\Delta \zeta] .
$$

We introduce similarly the sequence $\left(w_{n}\right)_{n}$, and prove that it converges to a solution $w$ of 3.20 . Now, we claim that $\Gamma \equiv 0$. In this case 3.21 is replaced by

$$
\Delta \Theta\left(T, w^{0}\right) \in[(j-2) \pi+\Delta \zeta,(j-1) \pi+\Delta \zeta] .
$$

Then, we introduce $\bar{t}>T$ such that $\Delta \Theta\left(\bar{t}, w^{0}\right)=(j-1) \pi+\Delta \zeta$ and compute, since $-\frac{\theta^{\prime}(t)}{2 V_{1}(\cos \theta(t), \sin \theta(t))} \geq 1$, the validity of

$$
\int_{\zeta_{S}-((j-1) \pi+\Delta \zeta)}^{\zeta_{S}} \frac{d \theta}{2 V_{1}(\cos \theta, \sin \theta)}=b_{j}^{1} \geq \bar{t}
$$

bringing us to the conclusion $T=b_{j}^{1} \geq \bar{t} \geq T$, hence $T=\bar{t}$.

Once proved that $w$ cover the angle $(j-1) \pi+\Delta \zeta$ in the interval $[0, T]$, we can introduced the polar coordinates induced by $\varphi_{V_{1}}$ :

$$
w(t)=r(t) \varphi_{V_{1}}(t+\omega(t)) .
$$

A standard computation provides

$$
\begin{gathered}
r^{\prime}(t)=-r(t) \Gamma(t)\left\langle\nabla V_{2}\left(\varphi_{V_{1}}(t+\omega(t))\right) \mid \varphi_{V_{1}}^{\prime}(t+\omega(t))\right\rangle, \\
\omega^{\prime}(t)=\Gamma(t)\left(2 V_{2}\left(\varphi_{V_{1}}(t+\omega(t))\right)-1\right) .
\end{gathered}
$$

Again $\omega(0)=\omega(T)=\tau_{0, V_{1}}$ and we can similarly conclude that $\Gamma \equiv 0$ almost everywhere in $[0, T]$, thus obtaining $w(t)=C \varphi_{V_{1}}\left(t+\tau_{0, V_{1}}\right)$ for a suitable positive constant $C$. Then, introducing standard polar coordinates for the sequence $\left(w_{n}\right)_{n}$, we can prove that

$$
\int_{\theta_{n}(T)}^{\theta_{n}(0)} \frac{d \theta}{2 V_{1}(\cos \theta, \sin \theta)} \leq T
$$

for large indexes $n$. Similarly as above we can compute

$$
\int_{\theta_{n}(T)}^{\theta_{n}(0)} \frac{d \theta}{2 V_{1}(\cos \theta, \sin \theta)}=T+\int_{0}^{T} \frac{\left\langle\mathcal{G}\left(t, z_{n}(t)\right) \mid z_{n}(t)\right\rangle}{2 V_{1}\left(z_{n}(t)\right)}=: T+\mathcal{X}_{n}
$$


where now $\mathcal{G}(t, z)=g(t, z)+p(t, z)-\nabla V_{1}(z)$ and $\mathcal{X}_{n} \leq 0$ for every $n$. Parametrizing the solutions $z_{n}$ in the polar coordinates induced by $\varphi_{V_{1}}$, i.e. $z_{n}(t)=$ $r_{n}(t) \varphi_{V_{1}}\left(t+\omega_{n}(t)\right)$ we obtain

$$
\begin{aligned}
0 & \geq \liminf _{n \rightarrow+\infty}\left\|z_{n}\right\|_{\infty} \mathcal{X}_{n} \\
& \geq \int_{0}^{T} \liminf _{n \rightarrow+\infty} \frac{\left\langle\mathcal{G}\left(t, r_{n}(t) \varphi_{V_{1}}\left(t+\omega_{n}(t)\right)\right) \mid \varphi_{V_{1}}\left(t+\omega_{n}(t)\right)\right\rangle}{\frac{r_{n}(t)}{\left\|z_{n}\right\|_{\infty}}} d t \\
& \geq \frac{1}{C} \int_{0}^{T} \liminf _{(\lambda, \omega) \rightarrow\left(+\infty, \tau_{0}, V_{1}\right)}\left\langle\mathcal{G}\left(t, \lambda \varphi_{V_{1}}(t+\omega)\right) \mid \varphi_{V_{1}}(t+\omega)\right\rangle d t .
\end{aligned}
$$

Finally, the last inequality, using (3.13), can be rewritten as $\mathcal{J}^{-}\left(\tau_{0, V_{2}}\right) \leq 0$ which contradicts Assumption 3.5

This prove the case (R4).

The modification needed to prove the case (R5) from the previous situation, are similar to the ones provided when $(\bar{R} 2)$ holds.

\subsection{Double resonance}

In this section we consider the case $T \in \partial \widetilde{\mathcal{I}} \backslash \partial \mathcal{I}$, where $\mathcal{I}$ and $\widetilde{\mathcal{I}}$ were introduced in 2.19) and 2.20). In particular, $T=\beta_{j-1}=\alpha_{j}$ for a certain $j \in \mathbb{N}$.

In such a situation one among the alternatives $(\mathrm{R} 1)-(\mathrm{R} 3)$ is fulfilled and one among (R4)-(R6). We thus need to introduce a double Landesman-Lazer type of condition in order to find solutions to (3.1), i.e. the validity of two of the requirements in Assumption 3.5 is necessary.

Theorem 3.8 (Double resonance). Consider the problem (3.3), where $g$ satisfies Assumption 2.1 and p satisfies Assumption 3.1. If $T \in \partial \widetilde{\mathcal{I}} \backslash \partial \mathcal{I}$, then there exists at least one solution of (3.1) provided that Landesman-Lazer Assumptions 3.5 are fulfilled.

Proof. We focus our attention on the situation which presents all the difficulties: we thus assume that both $(\mathrm{R} 3)$ and $(\mathrm{R} 6)$ hold.

Let us consider $z_{0} \in l_{S}=l_{S}^{1} \cup l_{S}^{2}$ and the solution $\Phi\left(\cdot, z_{0}\right)$ of (3.3). If $\left|z_{0}\right|$ is sufficiently large, we have (no more (3.16) or 3.29 , but)

$$
\Delta \Theta\left(T, z_{0}\right) \in((j-2) \pi+\Delta \zeta,(j+1) \pi+\Delta \zeta) .
$$

We need to avoid the situation

$$
\Delta \Theta\left(T, z_{0}\right) \in((j-2) \pi+\Delta \zeta,(j-1) \pi+\Delta \zeta] \cup[j \pi+\Delta \zeta,(j+1) \pi+\Delta \zeta) .
$$

for every $z_{0} \in l_{S}$ such that $\left|z_{0}\right|$ is sufficiently large. 
We assume the existence of four diverging sequences: $\left(z_{n, 1}^{0}\right)_{n} \subset \ell_{S}^{1},\left(z_{n, 2}^{0}\right)_{n} \subset$ $\ell_{S}^{1},\left(z_{n, 3}^{0}\right)_{n} \subset \ell_{S}^{2},\left(z_{n, 4}^{0}\right)_{n} \subset \ell_{S}^{2}$, such that

$$
\begin{aligned}
& \Delta \Theta\left(T, z_{n, 1}^{0}\right) \in((j-2) \pi+\Delta \zeta,(j-1) \pi+\Delta \zeta], \\
& \Delta \Theta\left(T, z_{n, 2}^{0}\right) \in[j \pi+\Delta \zeta,(j+1) \pi+\Delta \zeta), \\
& \Delta \Theta\left(T, z_{n, 3}^{0}\right) \in((j-2) \pi+\Delta \zeta,(j-1) \pi+\Delta \zeta], \\
& \Delta \Theta\left(T, z_{n, 4}^{0}\right) \in[j \pi+\Delta \zeta,(j+1) \pi+\Delta \zeta) .
\end{aligned}
$$

For all the four sequences, thanks to the validity of the Landesman-Lazer Assumption 3.5 adapting the proof of Theorem 3.7 we will get a contradiction.

In this way, we obtain the existence of $R>0$ such that, if $z_{0} \in l_{S}^{2} \cup l_{S}^{1}$ and $\left|z_{0}\right|>R$ then the solution $\Phi\left(\cdot, z_{0}\right)$ of 3.3 satisfies

$$
\Delta \Theta\left(T, z_{0}\right) \in((j-1) \pi+\Delta \zeta, j \pi+\Delta \zeta) .
$$

Hence, we are in the situation of Remark 3.4 and we can conclude as in the proof of Theorem 3.2 .

\section{Application to asymmetric nonlinearities}

In this section we focus our attention on scalar differential equations

$$
x^{\prime \prime}+f(t, x)=0,
$$

where $f$ is a continuous function satisfying

$$
\begin{aligned}
& 0<\nu_{1} \leq \liminf _{x \rightarrow-\infty} \frac{f(t, x)}{x} \leq \limsup _{x \rightarrow-\infty} \frac{f(t, x)}{x} \leq \nu_{2}, \\
& 0<\mu_{1} \leq \liminf _{x \rightarrow+\infty} \frac{f(t, x)}{x} \leq \limsup _{x \rightarrow+\infty} \frac{f(t, x)}{x} \leq \mu_{2},
\end{aligned}
$$

uniformly with respect to $t$ (we assume $f$ to be continuous just to simplify the argument).

We address the reader to [1, Section 3] for a comparison with the case $\nu=$ $\nu_{1}=\nu_{2}, \mu=\mu_{1}=\mu_{2}$. In this section we extend the results presented there. In particular, we will focus our attention only on the Dirichlet problems. The case of problems with Neumann boundary conditions or mixed boundary conditions $x^{\prime}(0)=x(T)=0$, which are treated in [1] too, is left to the reader as an exercise, for briefness.

Setting $z(t)=\left(x(t), x^{\prime}(t)\right)$, we can write equation 4.1) in the form of a planar system as in (1.8) where $2 V_{i}=x^{\prime}(t)^{2}+\mu_{i}\left(x^{+}(t)\right)^{2}+\nu_{i}\left(x^{-}(t)\right)^{2}$. The planar system $J z^{\prime}=\nabla V_{i}(z)$, is nothing else but the asymmetric oscillator

$$
x^{\prime \prime}+\mu_{i} x^{+}-\nu_{i} x^{-}=0,
$$


and admits periodic solutions of period $\tau_{V_{i}}=\frac{\pi}{\sqrt{\mu_{i}}}+\frac{\pi}{\sqrt{\nu_{i}}}$ of the form $z(t)=$ $\left(x(t), x^{\prime}(t)\right)$ with $x(t)=C \phi_{\mu_{i}, \nu_{i}}\left(t+t_{0}\right)$ where $C \in \mathbb{R}^{+}, t_{0} \in \mathbb{R}$ and

$$
\phi_{\mu_{i}, \nu_{i}}(t):= \begin{cases}\frac{1}{\sqrt{\mu_{i}}} \sin \left(\sqrt{\mu_{i}} t\right) & t \in\left[0, \frac{\pi}{\sqrt{\mu_{i}}}\right], \\ \frac{1}{\sqrt{\nu_{i}}} \sin \left(\sqrt{\nu_{i}}\left(\frac{\pi}{\sqrt{\mu_{i}}}-t\right)\right) & t \in\left[\frac{\pi}{\sqrt{\mu_{i}}}, \frac{\pi}{\sqrt{\mu_{i}}}+\frac{\pi}{\sqrt{\nu_{i}}}\right] .\end{cases}
$$

Concerning the problem with Dirichlet boundary conditions, we can compute the constants introduced in Section 2.1.

$$
\tau_{0, V_{i}}=\sigma_{1, V_{i}}=\sigma_{2, V_{i}}=0, \quad \tau_{1, V_{i}}=\frac{\pi}{\sqrt{\mu}_{i}}, \quad \tau_{2, V_{i}}=\frac{\pi}{\sqrt{\nu}_{i}} .
$$

In particular, the scalar problem

$$
\left\{\begin{array}{l}
x^{\prime \prime}+\mu_{i} x^{+}-\nu_{i} x^{-}=0, \\
x(0)=0=x(T),
\end{array}\right.
$$

has nontrivial solutions if one of the following identities holds for a certain $k \in \mathbb{N}$ :

$$
\begin{aligned}
& T=\alpha_{i, k}:=\pi\left[(k+1) \frac{1}{\sqrt{\mu_{i}}}+k \frac{1}{\sqrt{\nu_{i}}}\right], \\
& T=\beta_{i, k}:=\pi\left[k \frac{1}{\sqrt{\mu_{i}}}+(k+1) \frac{1}{\sqrt{\nu_{i}}}\right], \\
& T=\gamma_{i, k}:=\pi(k+1)\left[\frac{1}{\sqrt{\mu_{i}}}+\frac{1}{\sqrt{\nu_{i}}}\right] .
\end{aligned}
$$

They are indeed equivalent to 2.10)-2.13. In particular, $\phi_{\mu_{i}, \nu_{i}}$ solves 4.4 when 4.5 or 4.7 holds, while

$$
\psi_{\mu_{i}, \nu_{i}}(t):=\phi_{\mu_{i}, \nu_{i}}\left(t+\pi / \sqrt{\mu_{i}}\right)
$$

solves it when 4.6 or 4.7 holds.

Let us now focus on the Dirichlet problem

$$
\left\{\begin{array}{l}
x^{\prime \prime}+f(t, x)=0, \\
x(0)=0=x(T),
\end{array}\right.
$$

where $f$ satisfies 4.2 and 4.3 . The resonance set $\mathcal{I}$ in 2.19 is now

$$
\mathcal{I}=\bigcup_{k>0}\left[\min \left\{\alpha_{2, k}, \beta_{2, k}\right\}, \max \left\{\alpha_{1, k}, \beta_{1, k}\right\}\right] \cup\left[\gamma_{2, k}, \gamma_{1, k}\right] .
$$

The resonance set $\mathcal{I}$ is useful when we want to investigate resonance phenomena when $T$ varies and the constants $\mu_{1}, \mu_{2}, \nu_{1}, \nu_{2}$ are fixed a priori. On the contrary, 


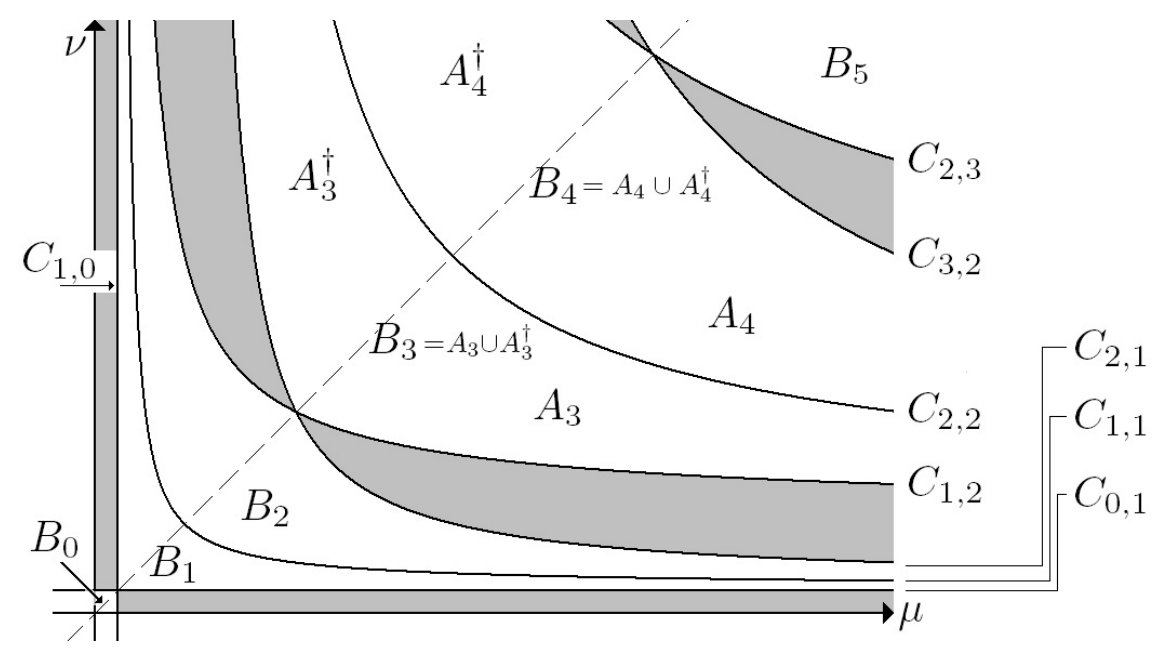

Figure 3: The Dancer-Fučík spectrum for the Dirichlet problem. The open rectangle $\mathcal{R}=\left(\mu_{1}, \mu_{2}\right) \times\left(\nu_{1}, \nu_{2}\right)$ can't intersect the forbidden region in grey and the spectrum lines $C_{a, b}$. Besides, the vertices of $\mathcal{R}$ can intersect the spectrum.

fixing $T$, we can study resonance when the other constants change using the set $\Sigma_{D}$ known as the Dancer-Fučík spectrum associated to (4.4). We recall that, for a fixed $T$, the set $\Sigma_{D}$ collects all the couples $(\mu, \nu)$ in the first quadrant $Q=\left(\mathbb{R}^{+}\right)^{2}$ satisfying one among 4.5 - -4.7$)$, and it consists of an infinite number of curves, see Figure 3 ,

$$
C_{a, b}=\left\{(\mu, \nu) \in Q \mid \frac{a}{\sqrt{\mu}}+\frac{b}{\sqrt{\nu}}=\frac{T}{\pi}\right\},
$$

where

$$
\begin{aligned}
(a, b) \in \Gamma:= & \left\{(a, b) \in \mathbb{N}^{2}|a+b>0,| a-b \mid \leq 1\right\} \\
& =\{(0,1),(1,0),(1,1),(1,2),(2,1),(2,2), \ldots\} .
\end{aligned}
$$

Notice that the curves $C_{k, k+1}$ and $C_{k+1, k}$ intersect in the point $\left(\lambda_{k}, \lambda_{k}\right)$, with $\lambda_{k}=(\pi(2 k+1) / T)^{2}$. Hence, we can write

$$
\Sigma_{D}=\bigcup_{(a, b) \in \Gamma} C_{a, b} .
$$

Let us introduce the sets

$$
A_{0}=\{(\mu, \nu) \in Q \mid \mu \geq \nu, \pi>T \sqrt{\mu}\}
$$

and, for every integer $j \geq 1$,

$$
A_{2 j-1}=\left\{(\mu, \nu) \in Q \mid \mu \geq \nu ; j\left(\frac{\pi}{\sqrt{\mu}}+\frac{\pi}{\sqrt{\nu}}\right)-\frac{\pi}{\sqrt{\mu}}<T<j\left(\frac{\pi}{\sqrt{\mu}}+\frac{\pi}{\sqrt{\nu}}\right)\right\},
$$




$$
A_{2 j}=\left\{(\mu, \nu) \in Q \mid \mu \geq \nu ; j\left(\frac{\pi}{\sqrt{\mu}}+\frac{\pi}{\sqrt{\nu}}\right)<T<j\left(\frac{\pi}{\sqrt{\mu}}+\frac{\pi}{\sqrt{\nu}}\right)+\frac{\pi}{\sqrt{\mu}}\right\} .
$$

Then we define $A_{k}^{\dagger}=\left\{(\mu, \nu) \in Q \mid(\nu, \mu) \in A_{k}\right\}$, which is the specular of $A_{k}$ with respect to the line $\mu=\nu$. Finally, define $B_{k}=A_{k} \cup A_{k}^{\dagger}$ and $B=\bigcup_{k \geq 0} B_{k}$, see Figure 3 , and the open rectangle

$$
\mathcal{R}=\left(\mu_{1}, \mu_{2}\right) \times\left(\nu_{1}, \nu_{2}\right) .
$$

Concerning the resonance phenomenon, we can summarize the possible situations verifying the position of the open rectangle $\mathcal{R}$ with respect to the DancerFučík spectrum as follows:

- Nonresonance: $\overline{\mathcal{R}} \subset B$,

- Resonance: $\mathcal{R} \subset B$ and $\overline{\mathcal{R}} \cap \Sigma_{D} \neq \varnothing$. In particular, in such a situation, one (simple resonance) or both (double resonance) the points $\left(\mu_{1}, \nu_{1}\right)$ and $\left(\mu_{2}, \nu_{2}\right)$ belongs to $\Sigma_{D}$.

In particular, we need to avoid the situation " $\mathcal{R}$ intersects $Q \backslash\left(B \cup \Sigma_{D}\right)$ " (roughly speaking, $\mathcal{R}$ can't intersect the grey-coloured region in Figure 3 ).

We now focus our attention on the Landesman-Lazer conditions we need to introduce. At first we define the values

$$
\begin{aligned}
& \mathcal{A}^{-}(\zeta):=\int_{\{\zeta>0\}}\left(\liminf _{x \rightarrow+\infty} f(t, x)-\mu_{1} x\right) \zeta(t) d t+ \\
& \int_{\{\zeta<0\}}\left(\limsup _{x \rightarrow-\infty} f(t, x)-\nu_{1} x\right) \zeta(t) d t, \\
& \mathcal{A}^{+}(\zeta):=\int_{\{\zeta>0\}}\left(\limsup _{x \rightarrow+\infty} f(t, x)-\mu_{2} x\right) \zeta(t) d t+ \\
& \int_{\{\zeta<0\}}\left(\liminf _{x \rightarrow-\infty} f(t, x)-\nu_{2} x\right) \zeta(t) d t .
\end{aligned}
$$

Collecting all the possibile situations in a unique statement we can summarize the Landesman-Lazer Assumption 3.5 for the Dirichlet problem 4.8 as follows.

Assumption 4.1. Assume $\mathcal{R} \subset B$ and

$$
\begin{array}{rll}
\text { if }\left(\mu_{1}, \nu_{1}\right) \in C_{h, h+1} \quad \text { then } & \mathcal{A}^{-}\left(\psi_{\mu_{1}, \nu_{1}}\right)>0, \\
\text { if }\left(\mu_{1}, \nu_{1}\right) \in C_{h+1, h} \quad \text { then } & \mathcal{A}^{-}\left(\phi_{\mu_{1}, \nu_{1}}\right)>0, \\
\text { if }\left(\mu_{1}, \nu_{1}\right) \in C_{h, h} \quad \text { then } & \mathcal{A}^{-}\left(\phi_{\mu_{1}, \nu_{1}}\right)>0 \text { and } \mathcal{A}^{-}\left(\psi_{\mu_{1}, \nu_{1}}\right)>0, \\
\text { if }\left(\mu_{2}, \nu_{2}\right) \in C_{k, k+1} \quad \text { then } & \mathcal{A}^{+}\left(\psi_{\mu_{2}, \nu_{2}}\right)<0 \\
\text { if }\left(\mu_{2}, \nu_{2}\right) \in C_{k+1, k} & \text { then } & \mathcal{A}^{+}\left(\phi_{\mu_{2}, \nu_{2}}\right)<0, \\
\text { if }\left(\mu_{2}, \nu_{2}\right) \in C_{k, k} & \text { then } & \mathcal{A}^{+}\left(\phi_{\mu_{2}, \nu_{2}}\right)<0 \text { and } \mathcal{A}^{+}\left(\psi_{\mu_{2}, \nu_{2}}\right)<0 .
\end{array}
$$


Remark 4.2. Concerning the previous assumption, if $\mathcal{R} \subset B_{2 j}$ for a positive integer $j$ then only the case $\left(\mu_{1}, \nu_{1}\right) \in C_{j, j}$ can hold, and similarly we can have $\left(\mu_{2}, \nu_{2}\right) \in C_{j, j+1}$ if $\mu \leq \nu$ or $\left(\mu_{2}, \nu_{2}\right) \in C_{j+1, j}$ if $\mu \geq \nu$. Similarly, if $\mathcal{R} \subset B_{2 j-1}$ for a positive integer $j$ then only the case $\left(\mu_{2}, \nu_{2}\right) \in C_{j, j}$ can hold, and similarly we can have $\left(\mu_{1}, \nu_{1}\right) \in C_{j, j-1}$ if $\mu \leq \nu$ or $\left(\mu_{1}, \nu_{1}\right) \in C_{j-1, j}$ if $\mu \geq \nu$.

Let us conlcude this paper with the existence theorem for the Dirichlet problem.

Theorem 4.3. Consider the problem 4.8) where the continuous function $f$ satisfies 4.2 and 4.3 .

If Assumption 4.1 holds, then there exists at least one solution of (4.8).

\section{Acknowledgements}

The author thanks Alessandro Fonda and Maurizio Garrione for the useful suggestions and discussions.

\section{References}

[1] A. Boscaggin and M. Garrione, Resonant Sturm-Liouville boundary value problems in differential systems in the plane, Z. Anal. Anwend. 35 (2016), $41-59$.

[2] E.N. Dancer, Boundary value problems for weakly nonlinear ordinary differential equations, Bull. Austral. Math. Soc. 15 (1976), 321-328.

[3] E.N. Dancer, Proof of the results in "Boundary value problems for weakly nonlinear ordinary differential equations", Rend. Istit. Mat. Univ. Trieste 42 (2010), 31-57.

[4] Y. Dong, On the solvability of asymptotically positively homogeneous equations with Sturm-Liouville boundary value conditions, Nonlinear Anal. 42 (2000), 1351-1363.

[5] P. Drábek and S. Invernizzi, On the periodic BVP for the forced Duffing equation with jumping nonlinearity, Nonlinear Anal. 10 (1986), 643-650.

[6] C. Fabry, Landesman-Lazer conditions for periodic boundary value problems with asymmetric nonlinearities, J. Differential Equations 116 (1995), 405-418.

[7] C. Fabry and A. Fonda, Periodic solutions of nonlinear differential equations with double resonance, Ann. Mat. Pura Appl. 157 (1990), 99-116.

[8] C. Fabry and A. Fonda, Nonlinear equations at resonance and generalized eigenvalue problems, Nonlinear Anal. 18 (1992), 427-444. 
[9] C. Fabry and A. Fonda, Periodic solutions of perturbed isochronous Hamiltonian systems at resonance, J. Differential Equations 116 (2005), 299-325

[10] C. Fabry and P. Habets, Periodic solutions of second order differential equations with superlinear asymmetric nonlinearities, Arch. Math. 60 (1993), $266-276$.

[11] A. Fonda, Positively homogeneous Hamiltonian systems in the plane, J. Differential Equations 200 (2004), 162-184.

[12] A. Fonda, Playing around resonance: an invitation to the search of periodic solutions for second order ordinary differential equations, Birkhäuser Advanced Texts Basler Lehrbücher, Springer International Publishing, 2016.

[13] A. Fonda and M. Garrione, Double resonance with Landesman-Lazer conditions for planar systems of ordinary differential equations, J. Differential Equations 250 (2011), 1052-1082.

[14] A. Fonda and M. Garrione, Generalized Sturm-Liouville boundary conditions for first order differential systems in the plane, Topological Methods Nonlinear Anal. 42 (2013), 293-325.

[15] A. Fonda and J. Mawhin, Planar differential systems at resonance, Adv. Differential Equations 11 (2006), 1111-1133.

[16] A. Fonda and A. Sfecci, Periodic solutions of a system of coupled oscillators with one-sided superlinear retraction forces, Differential Integral Equations 25 (2012) , 993-1010.

[17] A. Fonda and A. Sfecci, Multiple periodic solutions of Hamiltonian systems confined in a box, Discrete Contin. Dynam. Syst. 37 (2017) 1425-1436.

[18] S. Fučík, Boundary value problems with jumping nonlinearities, Časopis Pěst. Mat. 101 (1976), 69-87.

[19] M. Henrard, Optimal integral criterion of nonresonance for asymptotically positively homogeneous equations with Sturm-Liouville boundary conditions, Acad. Roy. Belg. Cl. Sci. Mém. Collect. 818 (2000), 1-51.

[20] B.P. Rynne, The Fučík spectrum of general Sturm-Liouville problems, J. Differential Equations 161 (2000), 87-109.

[21] B.P. Rynne, Non-resonance conditions for semilinear Sturm-Liouville problems with jumping nonlinearities, J. Differential Equations 170 (2001), 215227.

[22] A. Sfecci, Positive periodic solutions for planar differential systems with repulsive singularities on the axes, J. Math. Anal. Appl. 415 (2014), 110120. 
[23] A. Sfecci, Double resonance for one-sided superlinear or singular nonlinearities, Ann. Mat. Pura Appl. 195 (2016), 2007-2025.

[24] A. Sfecci, Periodic impact motions at resonance of a particle bouncing on spheres and cylinders, Adv. Nonlinear Studies 17 (2017), 481-496.

[25] P. Tomiczek, Potential Landesman-Lazer type conditions and the Fučík spectrum, Electron. J. Differential Equations 2005 (2005), No. 94, pp. 112. 